EPJ Web of Conferences 116, 06010 (2016)

DOI: $10.1051 /$ epjconf/201611606010

(C) Owned by the authors, published by EDP Sciences, 2016

\title{
Development and performances of a high statistics PMT test facility
}

\author{
Carlos Maximiliano Mollo a on behalf of the KM3NeT Collaboration \\ INFN Napoli, Complesso Universitario di Monte Sant'Angelo, via Cintia, 80126 Napoli, Italy
}

\begin{abstract}
Since almost a century photomultipliers have been the main sensors for photon detection in nuclear and astro-particle physics experiments. In recent years the search for cosmic neutrinos gave birth to enormous size experiments (Antares, Kamiokande, SuperKamiokande, etc.) and even kilometric scale experiments as ICECUBE and the future $\mathrm{KM} 3 \mathrm{NeT}$. A very large volume neutrino telescope like KM3NeT requires several hundreds of thousands photomultipliers. The performance of the telescope strictly depends on the performance of each PMT. For this reason, it is mandatory to measure the characteristics of each single sensor. The characterization of a PMT normally requires more than 8 hours mostly due to the darkening step. This means that it is not feasible to measure the parameters of each PMT of a neutrino telescope without a system able to test more than one PMT simultaneously. For this application, we have designed, developed and realized a system able to measure the main characteristics of 623 -inch photomultipliers simultaneously. Two measurement sessions per day are possible. In this work, we describe the design constraints and how they have been satisfied. Finally, we show the performance of the system and the first results coming from the test of few thousand tested PMTs.
\end{abstract}

\section{Introduction}

The KM3NeT Collaboration [1] is aimed at realize a telescope for the detection of high energy astrophysical neutrinos by reconstructing the trajectory of the muon generated by the neutrino interaction with the Earth. Digital optical modules (DOM) will detect the Cerenkov light generated by muons in the seawater, where the telescope will be placed. Each DOM is equipped with 31 3-inch PMTs manufactured by Hamamatsu [2]. Approximately 115 strings or Detector Units (DUs) will constitute the first so-called building block of the telescope (3 building blocks will constitute the complete telescope), each DU has 18 DOMs then 64170 PMTs will be needed to complete the first building block. The knowledge of all principal parameters of each PMT is essential in order to have a good calibration of the telescope. In addition, it is necessary to determine the optimal high voltage of each PMT in order to have almost the same gain for all photodetectors. The measurement of the characteristics of 64170 PMTs could be a bottleneck in the telescope realization. Therefore, a system capable to measure several PMTs in parallel is mandatory. The realization of such a system has several challenges: timing synchronization and

\footnotetext{
a e-mail: maximil@na.infn.it
}

This is an Open Access article distributed under the terms of the Creative Commons Attribution License 4.0, which permits unrestricted use, distribution, and reproduction in any medium, provided the original work is properly cited. 


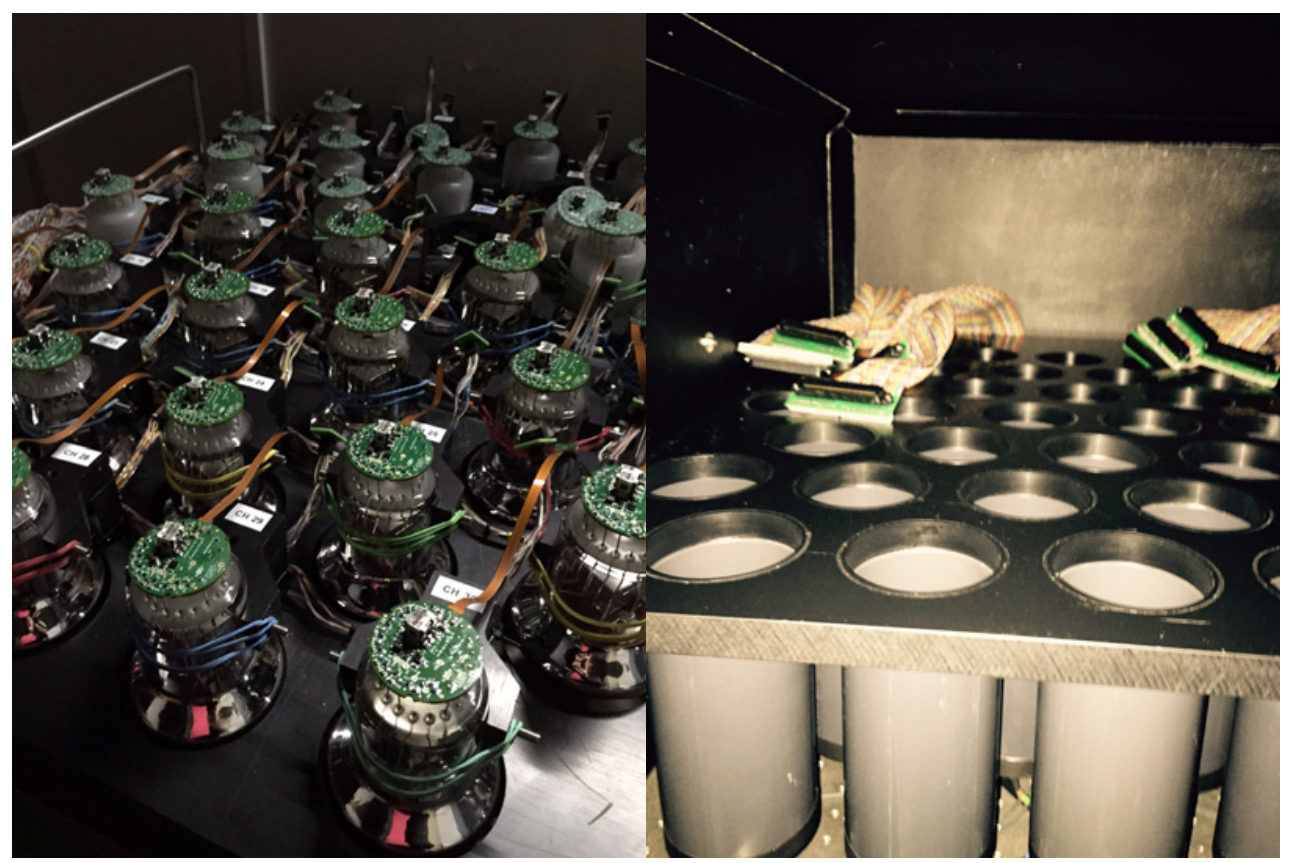

Figure 1. Left: PMTs on a tray ready to be tested; right: dark cylinders and cable extension connectors inside the Dark Box.

uniformity of a laser light needed to illuminate the PMTs for time calibration measurements. For the test facility we decided to use the same DAQ electronics of the DOMs. As said before each DOM electronics is able to handle the signals of 31 PMTs. Using two complete DOM electronics and synchronizing them by using a White Rabbit Switch we are able to test 62 PMTs in parallel.

\section{Mechanical and optical setup}

In order to test 62 PMTs in parallel we need to optically insulate a PMT with respect the others using dark cylinders (Fig. 1 right), in this way timing measurements like TTS (Transit Time Spread) or spurious pulses can't be corrupted by light coming from the near or even distant PMT test docks. At the base of each cylinder there is an opal diffusing glass disc that ensures a near uniform distribution of the light illuminating each photocathode uniformly even at single photon-electron condition. This is very important for TTS measurements.

In order to perform timing measurements like TTS, TT and spurious pulses, we have used one single light source, a picosecond LASER source from PiLas with excellent timing performances. The wavelength is $470 \mathrm{~nm}$ and the jitter between the trigger signal and the light pulse is less than $40 \mathrm{ps}$. To illuminate all 62 PMTs concurrently we have adopted an optical splitter 1 to 70 . It shows a good power output distribution as shown in Fig. 2.

The PMTs are placed in a tray of $57 \mathrm{~cm}$ by $66 \mathrm{~cm}$. Each tray has 4 pins that ensure the correct distance from the table when positioning the PMTs and also are used to guide the tray insertion into the Dark Box. 

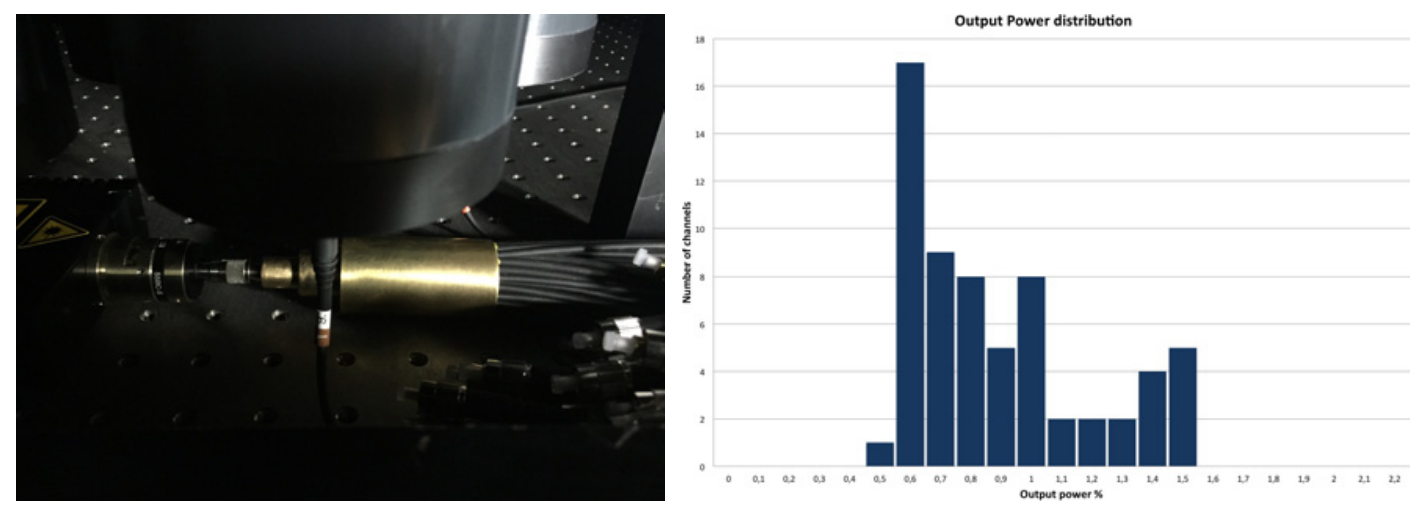

Figure 2. The optical splitter inside the Dark Box (left). The splitter output power distribution (right), all power outputs are within $0.5 \%$ and $1.5 \%$ of the input power.

\section{Electronics}

The DAQ electronics is based on the KM3NeT Central Logic Board (CLB). The CLB is the main logic board of the KM3NeT DOM. It is directly connected with a Power Conversion Board which provides all the needed rails for the board $(1.0 \mathrm{~V}, 1.8 \mathrm{~V}, 2.5 \mathrm{~V}, 3.3 \mathrm{~V}, 5.0 \mathrm{~V})$. The purpose of the CLB is to manage all the instrumentation present inside the DOM, acquire the data from the 31 PMTs and from the acoustic device, compress the data into User Datagram Protocol (UDP) packets and send them through the optical line. A sub-ns time synchronization level between all the DOMs is achieved by using White Rabbit switchs. The CLB is based on a Xilinx Kintex 7 FPGA, which is connected to all the rest of the board, including in particular:

- 1 Small Form-factor Pluggable (SFP) connector for laser transceivers for the optical communication;

- 2 custom Octopus connectors for the PMTs signals;

- Tunable oscillators (White Rabbit compliant);

- Embedded instrumentations (temperature and humidity sensor, tilt \& compass);

- External connectors (for Nanobeacon LED, acoustic devices, expansion boards).

For the purposes of the PMT test facility we only use the White Rabbit gear and acquire the data from PMTs. In order to record the data from 62 PMTs simultaneously we used two CLBs synchronized using a White Rabbit switch. The laser pulse emission is synchronized and syntonized to the CLB common clock and absolute time allowing for the measurement of PMT transit time (TT) [4].

\section{DAQ software}

The most part of the software tools were taken or re-adapted from the KM3NeT DAQ system, which was still in development phase (www.km3net.org/TDR/TDRKM3NeT.pdf). The software chain, together with providing fast analysis of the PMTs characteristics, has to be interfaced with the KM3NeT Database System. This step is necessary to: retrieve in real time information on the PMT assembly (e.g. serial number, vendor's HV setting, test and integration history); store the results of the calibration and make them available to the DOM integration teams for cross checks and further analysis.

Two data types are analysed: monitoring and optical data. The first one contains the number of pulses from each channel in the time-slice; the second contains arrival time (timestamp) and ToT (Time over Threshold) of each hit [5]. 


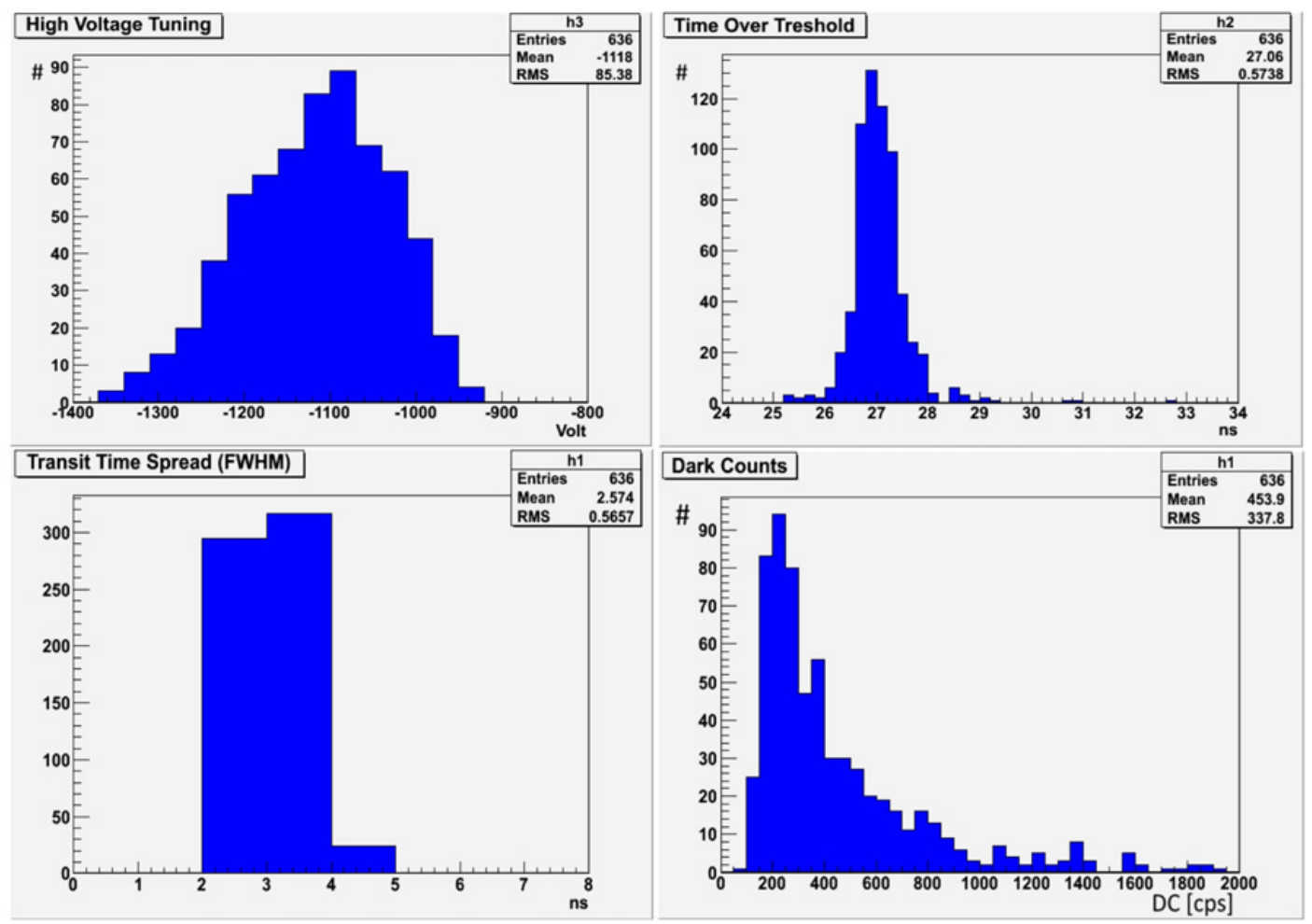

Figure 3. Distributions of the results of the test of 636 PMTs. High voltage tuning distribution (top-left), ToT distribution (top-right), Transit time spread distribution (bottom-left) and Dark counts distribution (bottom-right).

The data analysis code is as a $\mathrm{C}++$ program interfaced with ROOT libraries to create ROOT files and text output with the final plots and values. XML file creator is a ROOT script which creates a file with the PMT test results to be uploaded to the database. Also it generates a separate PDF test sheet for each PMT with the test results.

\section{Measurement results and conclusions}

The full test and calibration procedure for 62 PMTs takes about 12 hours including the analysis of all the data acquired. During the test almost all the PMT characteristics are measured. At the end of a test session all raw data and processed data are recorded into the KM3NeT Data Base in order to be available during the DOM production. The distributions of Dark Counts, ToT, TTS, and Tuned HV for about 600 PMTs are shown in Fig. 3.

The Dark Box system allows to test up to 124 PMTs per day, with usual methods the test of such number of PMTs would require weeks or months. The performance in terms of reliability were tested by comparing the test results obtained with the Dark Box with data from Hamamatsu. To speed up the sorting of PMTs to the integration sites it was adopted a triage (RED, YELLOW, GREEN) that allows a quick selection of those PMTs ready to be integrated (within the specifications) "GREEN". The PMTs that present characteristics out of specifications (for example: dark counts over $3000 \mathrm{cps}$, prepulses more than $1 \%$, etc.) are labelled "RED". The PMTs with characteristics slightly above the specifications are labelled "YELLOW" (for example: dark counts within 2000-3000 cps). In conclusion, the system is in 
all aspects (amount of PMTs tested daily, reliability, safety, etc.) ready for the mass production of the KM3NeT neutrino telescope.

\section{References}

[1] KM3NeT collaboration - http://www.km3net.org/collaboration.php

[2] KM3NeT consortium, O. Kalekin et al. Photomultipliers for the KM3NeT optical modules. Nuclear Instruments and Methods in Physics Research A 695 (2012) 313-316

[3] LASER PiLas - http: //www .alsgmbh.com/

[4] White Rabbit Switch - http://www.ohwr.org/projects/white-rabbit/wiki/Switch

[5] V. Kulikovskiy, this conference 